\title{
Evaluation of Post Effect of Oscillating Magnetic Field Anti Malaria Therapy
}

\author{
Abajingin David Dele* \\ Department of Physics and Electronics, Adekunle Ajasin University Akungba-Akoko Ondo State, Nigeria \\ *Corresponding author: abajingindd@yahoo.com
}

Received September 29, 2014; Revised November 12, 2014; Accepted November 26, 2014

\begin{abstract}
This study evaluates the post effect of oscillating magnetic field (OSMF) of ( $55 \mathrm{H}_{\mathrm{z}}, 41 \mathrm{mT}, 6 \mathrm{~h} /$ day) anti malaria therapy on malaria infested rats (albino) of about three weeks old. The post effect evaluation involves the Packed Cell Volume (PCV), parasitamia or Parasitic Density (PD), superoxide dismutase (SOD), catalase (CAT), and the malondialdehyde (MDA) concentration of the Plasma and liver for the exposed rats after thirty days outside the OSMF environment. The PCV increased while the degree of parasitamia or Parasitic Density (PD) decreased over the period of investigation. Plasma and liver MDA concentration were statistically insignificantly reduced; plasma and liver SOD activities slightly increased, while plasma and liver CAT activities were significantly increased in OSMF exposed rats, $(\mathrm{P}<0.05)$. Oxidative challenges were experienced by the rats exposed to OSMF, oxidative stress did not occur.
\end{abstract}

Keywords: Oscillating magnetic field, plasmodium falciparium, oxidative stress, plasmodium ovale

Cite This Article: Abajingin David Dele, "Evaluation of Post Effect of Oscillating Magnetic Field Anti malaria Therapy.” American Journal of Medical Sciences and Medicine, vol. 2, no. 6 (2014): 126-130. doi: 10.12691/ajmsm-2-6-3.

\section{Introduction}

About 300 million acute cases of malaria are reported every year around the world, resulting in more than one million deaths, [1]. Approximately 90 percent of these deaths occur in Africa, mostly in young children. In Nigeria, statistics have shown that of the 60 percent of malaria cases, 30 percent results into childhood deaths; 25 percent results into children death and 11 percent of maternal deaths in one year, [2,3]. The financial loss from malaria in the form of treatment costs, prevention, and loss of man-hours has been estimated to be roughly one hundred and thirty two billion naira per year, [3].

Anti-malaria drug therapy and chemotherapy [4] are the major mode of treatment of malaria. As good as these modes of therapies are, there has been major setback in these modes of therapies in Nigeria, due to the use of adulterated anti-malaria drugs and in availability of chemotherapy service centers in both government and private hospitals. The malaria parasite (plasmodium) over the years has developed high resistance to each of the currently available unadulterated anti-malaria drugs as a result of persistence use by the host to the malaria parasite, $[5,6]$.

An oscillating magnetic field anti malaria therapy [7] was developed based on separate experimental studies [8,9], which showed that the Hemes left undestroyed by the malaria parasite produce a toxic environment within the blood to the plasmodium falciparium. The toxic effects of the free Hemes is reduced by plasmodium falciparium by binding the Hemes together into long nontoxic stacks, called Hemozoins, which act essentially as tiny bar magnets, [11]. The desired separation of the Hemozoins into Hemes has been observed to be possible by a reverse process of an oscillating magnetic field, [7].

In this study we evaluate the post effect of OSMF antimalaria therapy on the rats, thirty days after they have been assumed cured and taken out of the OSMF environment.

\section{Materials and Experimental methods}

\subsection{Materials}

The materials used for this study were; a solenoid, a wooden cage kept in the animal house of the Adekunle Ajasin University, cereal pellet and 12 Volts step down transformer. The 12 volts step down transformer was connected to an alternating current (AC) power source from which an AC current output of 5.50A was obtained, [7].

\subsection{Animal}

Ten albino rats, (five infested with malaria parasite, made up the experimental group), weighting an average of $65 \mathrm{~g}$ obtained from the Department of Parasitology, University College Hospital (UCH), Ibadan, Nigeria were used for this study. The ten rats were fed with cereal pellet and water, and housed in a wooden cage at room temperature (25-27) ${ }^{\circ} \mathrm{C}$ maintained in normoxic conditions under a $12 \mathrm{hrs}$ light dark cycle to acclimatize and to ensure full maturation of the malaria parasite for two 
weeks. Treatment of the animals was in accordance with the Principles of Laboratory Care (NIH Publication 85-23, revised 1985). Blood samples were taken from the two groups during the period of acclimatization. Other samples were taken from experimental rats during exposure to OSMF for fifteen consecutive days and after thirty days from the two groups when they have been taken from OSMF environment. These samples were put in anticoagulant bottles and taken to Adekunle Ajasin University Health Centre laboratory for parasite growth investigation.

\subsection{Evaluation Protocol}

The Packed cell Volume (PCV) of the blood sample was obtained using the expression

$$
P V C=\frac{\text { W.B.C }}{\text { Total Packed Cell }}
$$

where W.B.C is the white blood cell count.

The degrees of parasitamia or Parasitic Density (PD) were classified,,++++++ . Higher number of positive signs indicates more malaria parasite in the host animal.

The thoracic and the abdominal regions of the rats in the two groups were opened to expose the liver. Blood was obtained through the liver puncture and placed in heparinized blood bottles. The blood samples were centrifuged at 5,000 rpm for 5 mins; the plasma was then separated, tissue was homogenized in ice cold normal saline $(1: 4 \mathrm{w} / \mathrm{v})$, centrifuged and the supernatant were stored in the freezer until analysis. The lipid peroxidation for the liver was determined using thiobarbituric acid reactive substances (TBARS) method, while catalase and the superoxide dismutase (SOD) for this same tissue were respectively determined by the methods by Sinha [12] and Misra and Fridovich [13].

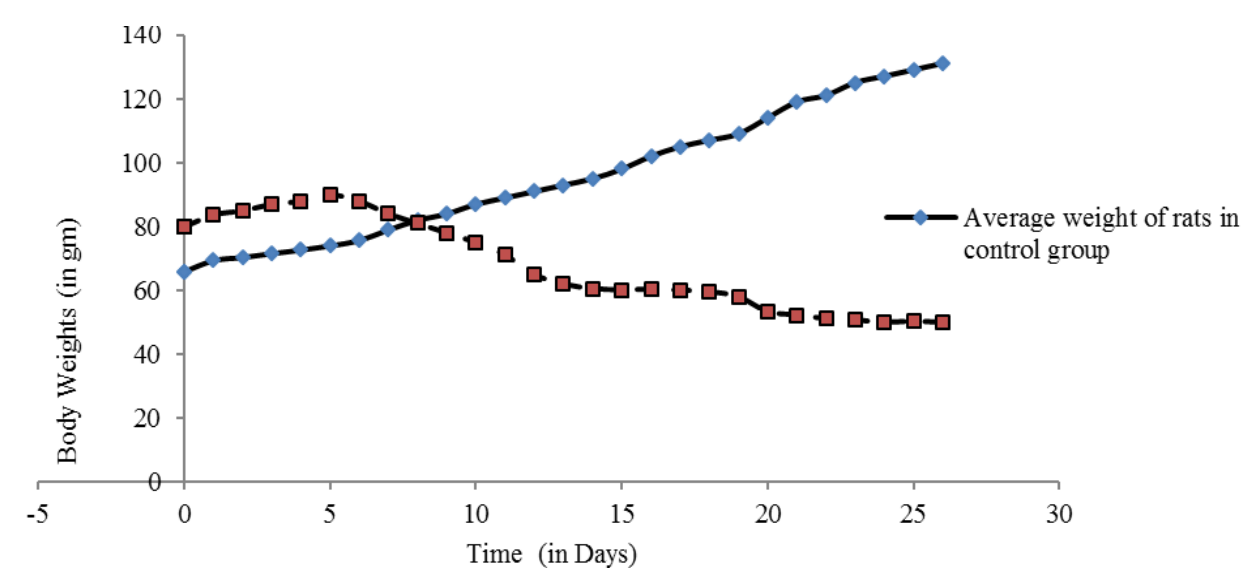

Figure 1. Variation of Body weight of the rats in both the control and the experimental groups with time

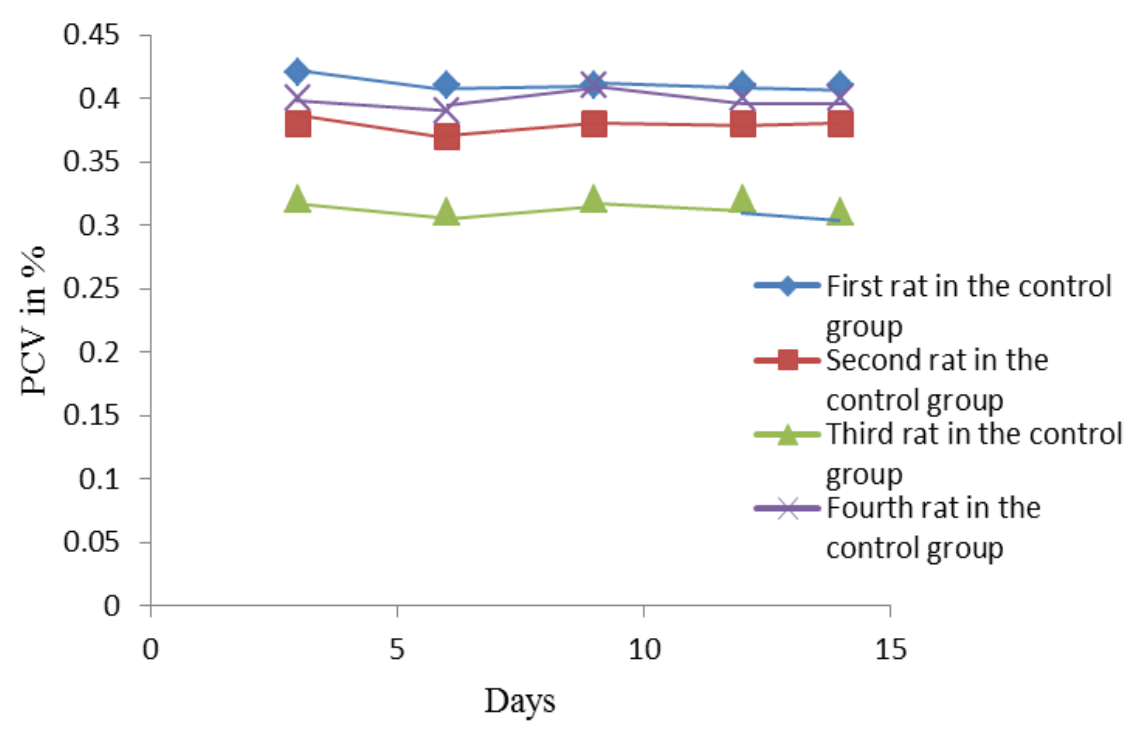

Figure 2. Graph of calculate PCV of the rats in the control group with time (Days)

\section{Results and Discussion}

The daily assessment of the weight of the rats showed that the body weight of the rats increased steadily for the rats in the control group. However, the body weight of the experimental group exposed to $\left(55 \mathrm{H}_{\mathrm{z}}, 41 \mathrm{mT}, 6 \mathrm{~h} /\right.$ day $)$ gradually increased for the first five days of acclimatization, but decreased appreciably from the seventh day of acclimatization period and throughout the period of the experiment, (Figure 1). These observations are similar to those of. Amara et al [14] and Lahbib et al [15] who reported a significant decreased in body weights of rats exposed to OSMF of ( $1 \mathrm{~h} /$ day at $128 \mathrm{mT})$ by the second week after thirty consecutive days of exposure and for fifteen consecutive days respectively. 
The variation of the PCV as calculated from equation (1) with time (in days), for the rats in the two groups are shown in Figure 2 and Figure 3. Figure 2 shows that the PCV of the rats in the control group was constant; this is an indication that these rats are free from malaria parasite while in Figure 3, the variation of the PCV of each rat in the experimental group shows a decrease with time. Since a decrease in PCV is always associated with increase in the degrees of parasitamia or Parasitic Density (PD), [16] we therefore asserts that the decrease in PCV as observed from the plots in Figure 3 is an indication of maturation of malaria parasites in the rats in the experimental group.

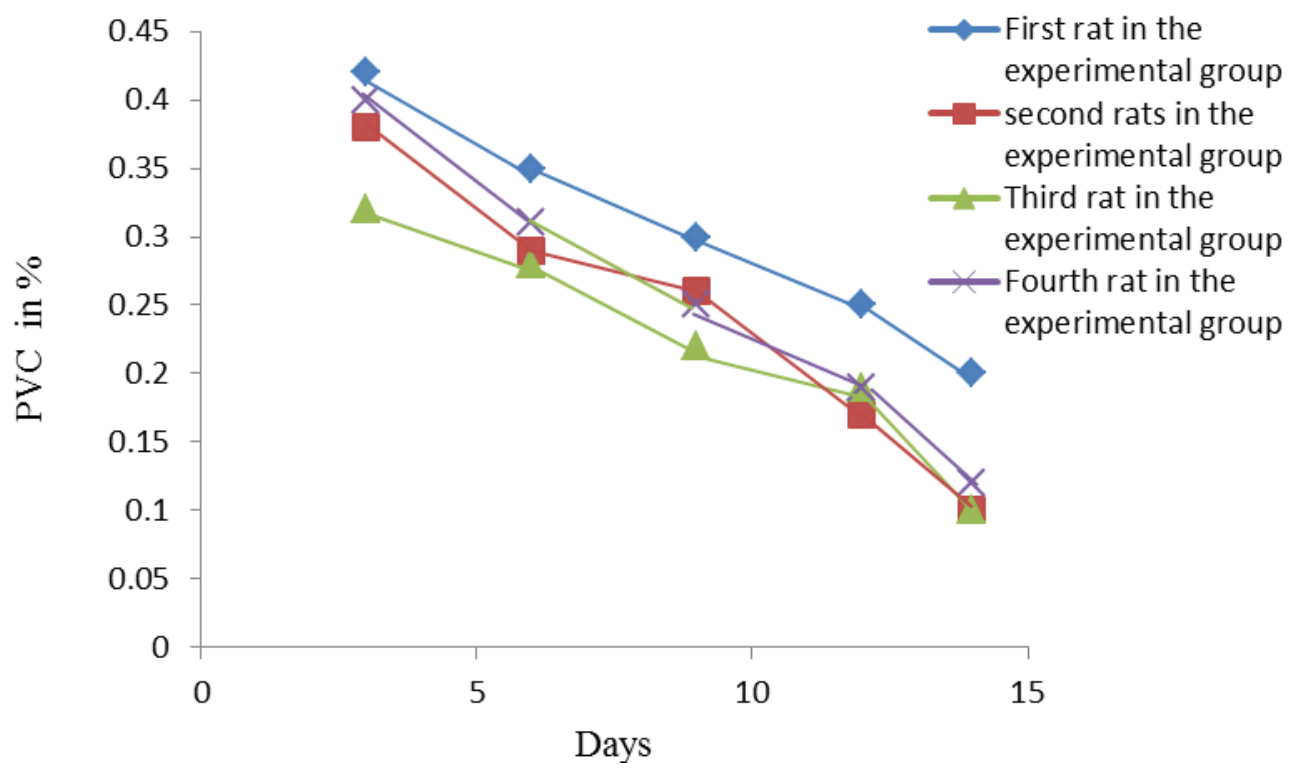

Figure 3. Graph of the PCV of rats in the experimental group with time (Days)

The difference in variation of the calculated PCV for each of the rats in the experimental group can be traced to the variation in the immunity of the individual rats to the malaria parasite, [16]

Figure 4 shows the variation of the PCV of the rats in the experimental group with time during exposure to OSMF. The inclination in the lines of variation of the PCV within the first seven days of exposure of the rats shows a rise in PCV with a subsequent decrease in the PD. This is an indication of delayed effect of the OSMF on the malaria infected rats. After the seventh day the OSMF became more effective as the variation of the PCV with time become appreciably constant within a range of about $42 \%$ to $40 \%$ for the first rat in downward trend. This same trend was observed for all the other rats in the experimental group.

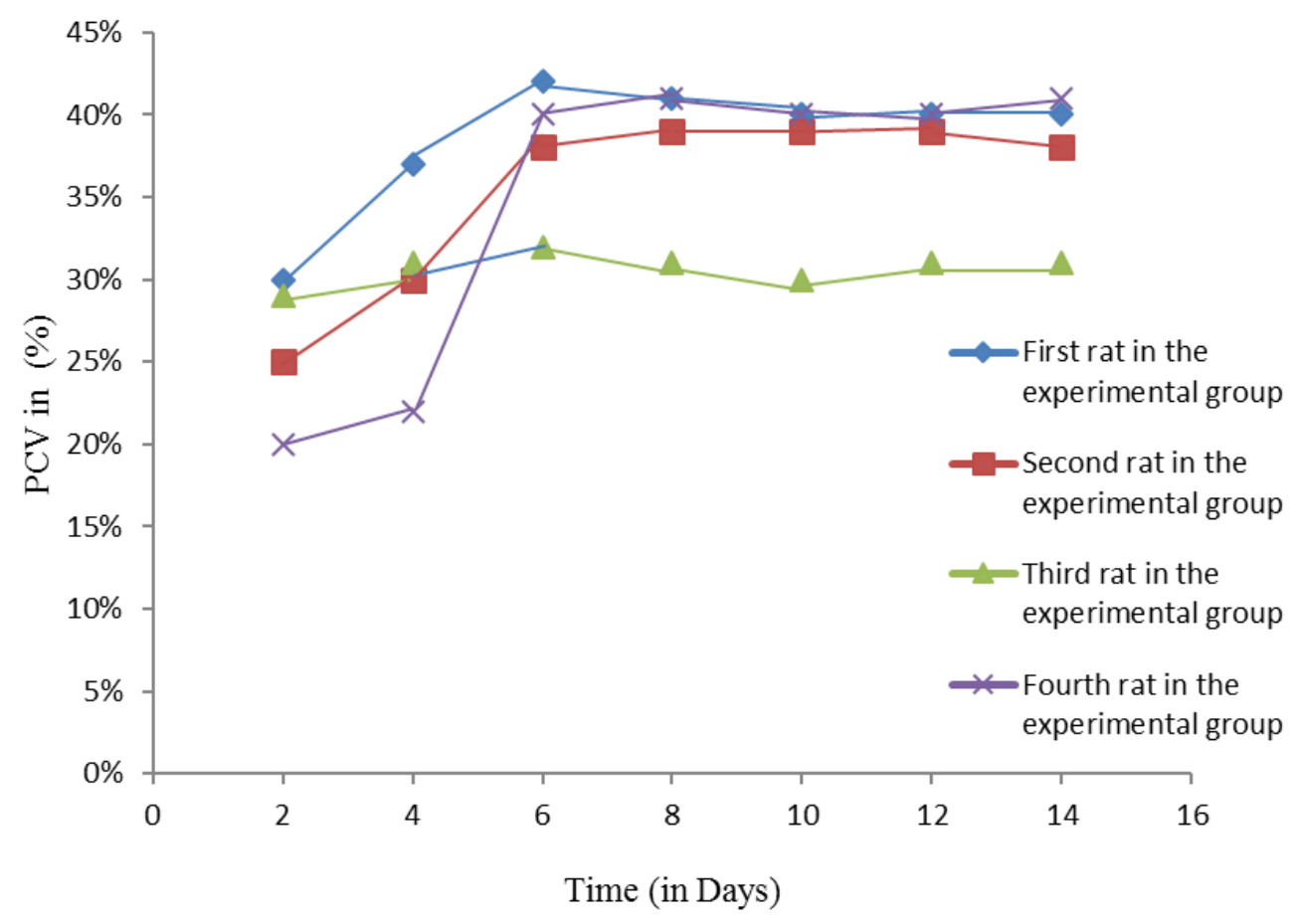

Figure 4. Variation of PCV experimental rats during exposure for fifteen days

Plasma and tissue superoxide dismutase (SOD) activities evaluated after thirty days out of OSMF environment revealed a slight decrease in plasma SOD activities of the exposed rats as compared with the control rats. The liver SOD activities of OSMF exposed rats were generally higher than control values, with. The results 
generated for the plasma SOD activities showed a slight decrease in the experimental rats while an insignificant increase in the SOD activities of the liver was observed, (Figure 5).

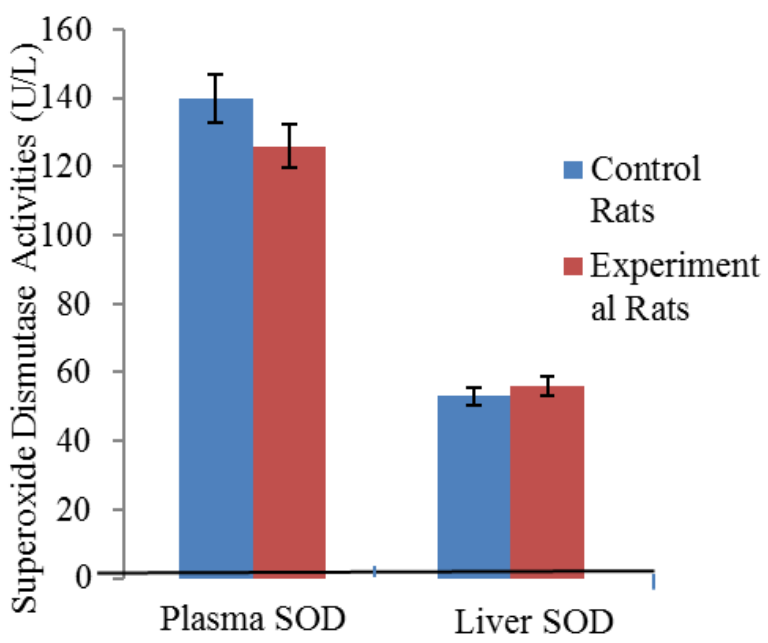

Figure 5. Effect of OSMF (55HZ, 41mT, 6h/day) on the plasma and liver superoxide dismutase (U/L) activities of rats. Data were obtained from plasma and liver homogenates at the end of 15 days exposure

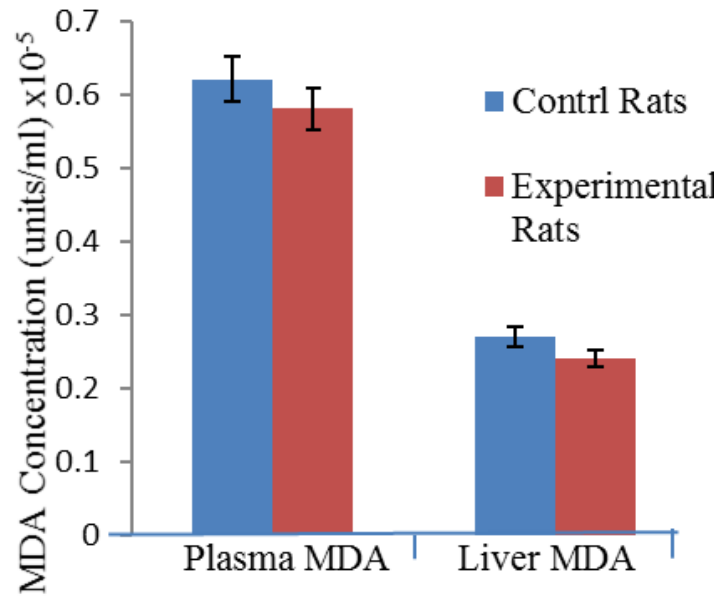

Figure 6. Effect of OSMF (55HZ, 41mT, 6h/day) on the plasma and liver MDA concentration (Units/ml x $10^{-5}$ ) activities of rats. Data were obtained from plasma and liver homogenates at the end of 15 days exposure

The plasma, liver, malondialdehyde (MDA) concentrations after thirty days out of OSMF environment rats were insignificantly lower than that of control rats (Figure 6).

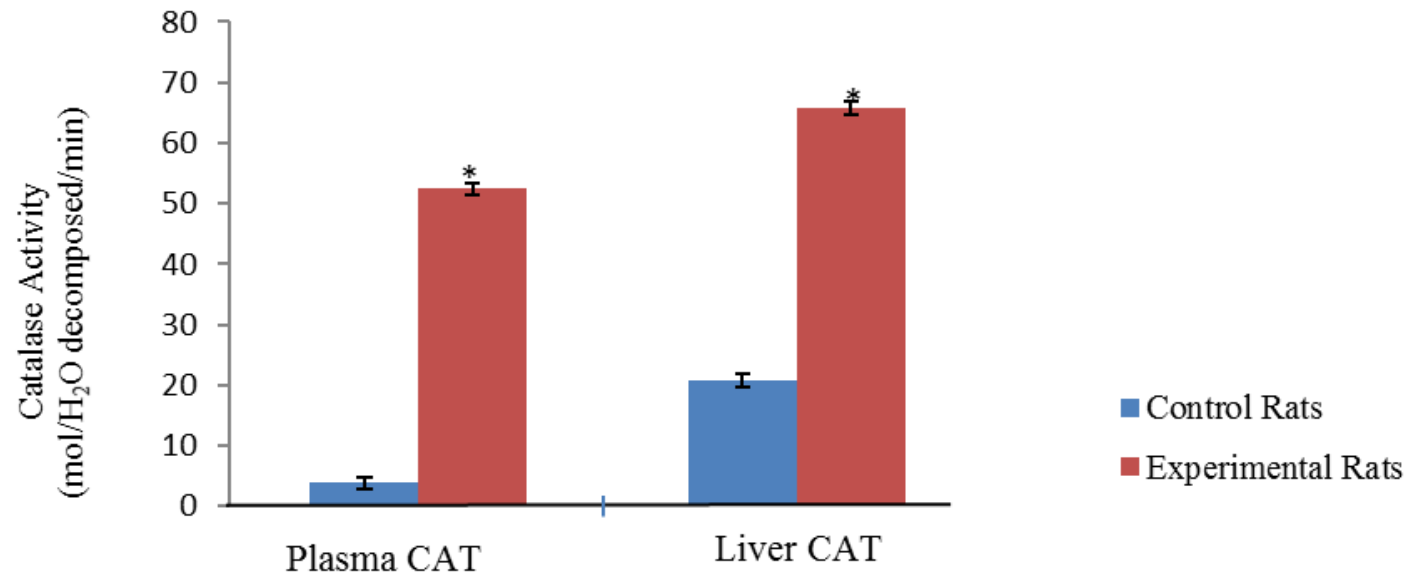

Figure 7. Effect of Oscillating magnetic field OSMF (55HZ, 41mT, 6h/day) on the plasma and liver catalase (mol $\mathrm{H}_{2} \mathrm{O}$ decomposition/min)v activities of rats. Data were obtained from plasma and liver homogenates at the end of 15 days exposure

After thirty days out of OSMF environment the plasma and liver catalase (CAT) activities of exposed rats were significantly higher than control value, $(\mathrm{p}<0.05)$ (Figure 7$)$.

\section{Conclusion}

Malaria transmission occurs by mosquito bites. Plasmodium sporozoites specifically invade the liver, where they develop into thousands of merozoites that initiate blood-stage infection and clinical malaria. This study was purposely designed to evaluate the post effect of OSMF anti malaria therapy on treated malaria infested rats. Since the liver is the major tissue where the malaria parasites develop, the combined adverse effect of the malaria parasite activities and the OSMF is central to this study with emphasis on the evaluation of the level of oxidative stress disturbance in this organ and in the plasma. This study showed that oscillating magnetic field is an effective anti-malaria therapy [7]. The increase in the liver SOD activities and the significant increases seen in plasma and liver CAT activities of OSMF exposed rats suggest that the rats though experienced oxidative challenges; no oxidative stress occurred. This result has a resemblance to those obtained by Politanski et al. [17] and Omonkhua and Abajingin [18], where the experimental rats were exposed to static magnetic field. Although this study has been limited to the effect of OSMF to the plasma and the liver, we conclude, based on the results from this study, that an OSMF is a better alternative anti malaria therapy to the anti-malaria drugs as it does not produce any significant adverse health effect on the user.

\section{Acknowledgement}

The author acknowledges Mr. Oluwaseun Ayodeji of Biochemistry Department University of Ibadan for helping to carry out the biochemistry analysis involved in this study and also the efforts of Dr. Omonkhua of college of Medicine, University of Benin, who ensures that the results of the biochemistry analysis were adequate. 


\section{References}

[1] Roll Back

Malaria http://nigeriamaps.org/?page_id=9\#sthash.sknDGcs5.dpuf

[2] Hay, S. Guerrac, T.A. Noor, A. Snow, R. (2004). The global distribution and population at risk of malaria; past, present and future. Lancet infect dis4 (6): 327-36.

[3] Feng, X. Joy, D. and May, J. (2003). Early Origin of Resent Expansions of Plasmodium Falciparum. Science 300 (5617) 318321.

[4] Nigerian Ministry of Health, http://nigeriamaps.org/?page_id=9\#sthash.sknDGcs5.dpuf

[5] Ronald Ross, (1928). Studies on Malaria Chemotherapy.

[6] Coxf, S. (2002). History of Human Parasitology. Microbial Rev 15 (4): 595-612.

[7] Akuyili, D. (2007)), Published in the Nation, July 10. Vol. 1 No. 0345, pp 3.

[8] Abajingin, D. D. Adegboye (2007). Oscillating magnetic field: an alternative anti-malaria therapy for malaria parasite infection. Science research annals; vol. 2 nos. 2, pp 126-130.

[9] Alex, S. R., (2001). Production of Hemozoins in Malaria Infested Rats. Unpublished Research Work by Research Team Work on Hemozoins at the Vanderbilt University, U.S.A.

[10] Henry A, Lai, H. (2000), Magnetic field may destroy malaria. Microbial Rev 9 (49): 268-269.

[11] Fairlamb A. H, Paul F, Warhurst D. C. A simple magnetic method for the purification of malarial pigment. Mol Biochem. Parasitol 1984; 12 : 307-312
[12] Sinha K. A (1972). Colorimetric assay of catalase. Anal. Biochem. 47: 389-394.

[13] Misra H.P, Fridovich I. (1972). The role of superoxide anion in the auto oxidation of epinephrine and a simple assay for superoxide dismutase. J. Biol. Chem. 247 (12): 3170-3175.

[14] Amara S, Abdelmelek H, Salem M. B. Abidi R, Sakly M. (2006), Effects of static magnetic field exposure on hematological and biochemical parameters in rats. Braz. Arch. Biol. Technol. 49 (6) 889-895.

[15] Lahbib A, Elferchichi M, Gghodbane S, Belguith H, Chater S, Sakly M, Abdelmelek H. (20ts. 10). Time-dependent effects of exposure to static magnetic field on glucose and lipid metabolism in rats. Gen. Physiol. Biophys. 29 (4): 390-395.

[16] Haussig, J. M.; Burgold, J.; Hafalla, J. C. R.; Matuschewski, K.; Kooij, T. W. A. (2014), Signatures of malaria vaccine efficacy in ageing murine immune memory. Parasite Immunology, Volume 36, Number 5, pp. 199-206 (8).

[17] Politanski, P., Rajkowska E, Pawlaczyk-Luscynska M, Dudarewicz A, Wiktorek-Smagur A, Sliwinska-Kowalsaska M, Zmyslony M. (2010). Static magnetic field affects oxidative stress in mouse cochlea. Int. J. Occup. Med. Environ. Health. 23 (4): 377-384.

[18] Omonkhua A. A, Abajingin D.D (2013). Effects of sub-chronic exposure to moderate intensity static magnetic field (SMF) on oxidative stress parameters of male rats. African Biochemistry Research. Vol. 7 (4), pp. 39-44. 